\title{
Junctional Adhesion Molecule C Mediates Leukocyte Adhesion to Rheumatoid Arthritis Synovium
}

\author{
Bradley J. Rabquer, ${ }^{1}$ Angela Pakozdi, ${ }^{1}$ James E. Michel, ${ }^{1}$ Bansari S. Gujar, ${ }^{1}$ \\ G. Kenneth Haines, III, ${ }^{2}$ Beat A. Imhof, ${ }^{3}$ and Alisa E. Koch ${ }^{4}$
}

Objective. Leukocyte infiltration into the rheumatoid arthritis (RA) synovium is a multistep process in which leukocytes leave the bloodstream and invade the synovial tissue (ST). Leukocyte transendothelial migration and adhesion to RA ST requires adhesion molecules on the surface of endothelial cells and RA ST fibroblasts. This study was undertaken to investigate the role of junctional adhesion molecule C (JAM-C) in mediating leukocyte recruitment and retention in the RA joint.

Methods. Immunohistologic analysis was performed on RA, osteoarthritis (OA), and normal ST samples to quantify JAM-C expression. Fibroblast JAM-C expression was also analyzed using Western blotting, cell surface enzyme-linked immunosorbent assay, and immunofluorescence. To determine the role of JAM-C in leukocyte retention in the RA synovium, in vitro and in situ adhesion assays and RA ST fibroblast transmigration assays were performed.

Results. JAM-C was highly expressed by RA ST lining cells, and its expression was increased in OA ST and RA ST endothelial cells compared with normal ST endothelial cells. JAM-C was also expressed on the surface of OA ST and RA ST fibroblasts. Furthermore,

Supported by the NIH (grants AI-40987 and AR-48267) and the Department of Veterans Affairs Office of Research and Development, Medical Research Service. Dr. Koch is recipient of the Frederick G. L. Huetwell and William D. Robinson, MD, Professorship in Rheumatology.

${ }^{1}$ Bradley J. Rabquer, PhD, Angela Pakozdi, MD, James E. Michel, BS, Bansari S. Gujar, MD: University of Michigan Medical School, Ann Arbor; ${ }^{2}$ G. Kenneth Haines, III, MD: Yale University, New Haven, Connecticut; ${ }^{3}$ Beat A. Imhof, PhD: Centre Medical Universitaire, Geneva, Switzerland; ${ }^{4}$ Alisa E. Koch, MD: VA Medical Service, Ann Arbor, and University of Michigan Medical School, Ann Arbor.

Address correspondence and reprint requests to Alisa E. Koch, MD, University of Michigan Medical School, Department of Medicine, Division of Rheumatology, 109 Zina Pitcher Drive, Ann Arbor, MI 48109. E-mail: aekoch@umich.edu.

Submitted for publication October 29, 2007; accepted in revised form June 6, 2008. we demonstrated that myeloid U937 cell adhesion to both OA ST and RA ST fibroblasts and to RA ST was dependent on JAM-C. U937 cell migration through an RA ST fibroblast monolayer was enhanced in the presence of neutralizing antibodies against JAM-C.

Conclusion. Our results highlight the novel role of JAM-C in recruiting and retaining leukocytes in the RA synovium and suggest that targeting JAM-C may be important in combating inflammatory diseases such as RA.

Rheumatoid arthritis (RA) is a chronic, systemic inflammatory disease that is characterized by erosive synovitis. The disease process is marked by neovascularization of the synovium, synovial lining cell hyperplasia, and inflammatory cell infiltration into the joint. Leukocyte extravasation into the synovium and synovial fluid is an active process mediated by cellular adhesion molecules (1). After traversing the endothelium, leukocytes adhere to other cells, including fibroblast synovial tissue (ST) lining cells, via specific adhesion molecule interactions (1). It has previously been shown that these RA ST fibroblasts express a number of different types of cellular adhesion molecules, including integrins (2), members of the immunoglobulin superfamily (3), and selectins (2). In addition, these cellular adhesion molecules act as costimulators, and therefore, these interactions may lead to activation of inflammatory cells and perpetuation of inflammation in the RA synovium.

Junctional adhesion molecules (JAMs) are a subfamily of the immunoglobulin superfamily that have been identified at tight junctions between epithelial cells and endothelial cells (ECs). To date, 5 members of the JAM family have been identified: JAM-A (4), JAM-B $(5,6)$, JAM-C (7,8), JAM4 (9), and JAM-like (JAML) (10). All JAMs have an extracellular domain with 2 Ig-like domains, a single transmembrane sequence, and a short cytoplasmic tail that contains adaptor molecule 
binding motifs (11), and a putative site for phosphorylation by protein kinase C $(12,13)$. JAM-A, JAM-B, and JAM-C have been shown to interact with each other in homophilic interactions, and heterophilic interactions between JAM-B and JAM-C have also been observed (7). In addition to binding interactions between family members, other receptors have been identified that bind JAMs. JAM-A has been shown to be a ligand for lymphocyte function-associated antigen 1 (14) on the surface of leukocytes and integrin $\alpha_{v} \beta_{3}$ in cis on the surface of ECs $(15,16)$. In addition, JAM-B is a ligand for very late activation antigen 4 on the surface of leukocytes (17), while JAM-C is a ligand for the leukocyte integrins Mac- 1 and $\alpha_{\mathrm{x}} \beta_{2}(18,19)$.

JAMs have previously been shown to be mediators in a number of disease processes, including disease models of inflammation. JAM-A has been implicated in monocyte and neutrophil infiltration in models of skin inflammation, experimental meningitis, cardiac ischemia-reperfusion, and hepatic ischemia-reperfusion $(4,20-22)$. In addition, both JAM-B and JAM-C play a role in the development of contact dermatitis by promoting leukocyte recruitment into the skin (23), while JAM-C mediates neutrophil transendothelial migration in murine models of acute peritonitis (24), pancreatitis (25), and pulmonary inflammation (26).

Recently, Palmer et al (27) showed that JAM-C plays a role in experimental arthritis. They demonstrated that administration of an anti-JAM-C antibody decreased the severity and delayed the onset of antigeninduced and serum-induced arthritis in mice, respectively. However, the mechanism by which JAM-C acts to mediate inflammation in RA has not been definitively shown. Therefore, we investigated the expression of JAM-C in RA synovium and on RA ST fibroblasts, and the role that JAM-C plays in leukocyte adhesion to RA ST fibroblasts and RA ST. We demonstrate that JAM-C plays a role in leukocyte adhesion to OA and RA synovium, suggesting that the reduced arthritis observed by the neutralization of JAM-C in animal models is due in part to reduced leukocyte adhesion in the joint.

\section{MATERIALS AND METHODS}

Antibodies and reagents. Recombinant human tumor necrosis factor $\alpha(\mathrm{TNF} \alpha)\left(\mathrm{ED}_{50} 0.02-0.05 \mathrm{ng} / \mathrm{ml}\right)$, stromal cell-derived factor $1 \alpha(\mathrm{SDF}-1 \alpha)$, and goat anti-human JAM-A, JAM-B, and JAM-C antibodies were purchased from R\&D Systems (Minneapolis, MN). Neutralizing rat antimouse/human JAM-C antibodies (H33 and F26) that were previously shown to recognize human JAM-C were used in all functional assays (8). Both antibodies were tested because of their differing properties; H33 inhibits both JAM-C homophilic interactions and JAM-B-JAM-C heterophilic interactions, while F26 inhibits JAM-C homophilic interactions only (28). Rabbit anti-human P-selectin and mouse antihuman Mac-1 were purchased from BD PharMingen (San Diego, CA). Rabbit anti-human actin antibody was obtained from Sigma (St. Louis, MO). Normal goat $\mathrm{IgG}$ and rat $\mathrm{IgG}$ were purchased from Santa Cruz Biotechnology (Santa Cruz, CA). Horseradish peroxidase-conjugated rabbit anti-goat antibody was obtained from Molecular Probes (Eugene, OR). Alexa Fluor 488 donkey anti-goat antibodies were purchased from Invitrogen (Carlsbad, CA).

Cell culture. RA and OA ST samples were obtained from patients undergoing arthroplasty or synovectomy. Normal ST samples were obtained at autopsy or from patients undergoing amputation, within 24 hours after death or limb removal. The protocol for obtaining patient consent and for the use of human tissue was approved by the Institutional Review Board at the University of Michigan. Fresh ST samples were minced and digested in a solution of Dispase, collagenase, and DNase to isolate fibroblasts (29). The cells were cultured in RPMI 1640 supplemented with $10 \%$ fetal bovine serum (FBS) in $175-\mathrm{mm}$ tissue culture flasks at $37^{\circ} \mathrm{C}$ in a humidified atmosphere with $5 \% \mathrm{CO}_{2}$. Cells were used at passage 5-9, at which time they were a homogeneous, $85-95 \%$ confluent population of fibroblasts. U937 cells (human monocytic lymphoma cell line) were cultured in RPMI supplemented with $10 \%$ FBS in $175-\mathrm{mm}$ tissue culture flasks at $37^{\circ} \mathrm{C}$ in a humidified atmosphere with $5 \% \mathrm{CO}_{2}$.

Immunohistologic analysis. Frozen ST samples obtained from RA, OA, and normal patients were cut into $\sim 5-\mu \mathrm{m}$ sections and stained using an immunoperoxidase method. Slides were fixed in cold acetone for 10 minutes. Following incubation with $3 \% \mathrm{H}_{2} \mathrm{O}_{2}$ for 30 minutes to block endogenous peroxidase, ST samples were blocked with $10 \%$ horse serum in phosphate buffered saline (PBS) at $37^{\circ} \mathrm{C}$ for 1 hour, and then incubated with goat anti-human JAM-C (10 $\mu \mathrm{g} / \mathrm{ml}$ ) or purified nonspecific goat $\mathrm{IgG}$ for 1 hour at room temperature in blocking buffer. The ST samples were washed with PBS, and a 1:100 dilution (in blocking buffer) of biotinylated horse anti-goat antibody was added and incubated for an additional 1 hour at room temperature. After washing, antibody binding was detected using a Vectastain ABC Elite kit (Vector, Burlingame, CA) and the chromogen 3,3'diaminobenzidine (DAB) (Vector). ST samples were counterstained with Gill's hematoxylin. Staining was evaluated by a pathologist $(\mathrm{GKH})$ who was blinded with regard to sample group. Slides were examined for cellular immunoreactivity, and cell types were distinguished based on their characteristic morphology. ST inflammation was assigned a score from 0 to 4 , depending on the degree of inflammatory cell invasion. ST vascularity was assigned a score from 1 to 4 , depending on the vessel density in the ST. The percentage of cells expressing JAMs was analyzed on synovial lining cells and on ECs.

Cell lysis and Western blotting. RA ST fibroblasts were lysed with lysis buffer $(175 \mu \mathrm{l})$ (Cell Lysis Buffer; Cell Signaling Technology, Beverly, MA) containing protease inhibitors. The protein concentration in each extract was determined using a Pierce bicinchoninic acid protein assay (Pierce, Rockford, IL). Sodium dodecyl sulfate-polyacrylamide gel electrophoresis was performed on cell lysates after equal 
protein loading, according to the method of Laemmli (30), and proteins were transferred onto a nitrocellulose membrane using a semi-dry transblotting apparatus (Bio-Rad, Hercules, CA). Blots were probed using goat polyclonal antibodies specific for JAM-C. The immunoblots were stripped and reprobed with rabbit anti- $\beta$-actin to verify equal loading. The immunoreactive protein bands were visualized by enhanced chemiluminescence (Amersham Biosciences, Piscataway, NJ). Densitometric analysis of the bands was performed using Un-Scan-It software, version 5.1 (Silk Scientific, Orem, UT).

Cell surface enzyme-linked immunosorbent assay (ELISA). RA ST fibroblasts $\left(1 \times 10^{5} /\right.$ well $)$ were seeded in 96-well plates (BD Falcon, Bedford, MA). Upon reaching confluence, following overnight serum starvation, RA ST fibroblasts were left untreated or were stimulated with TNF $\alpha$ $(25 \mathrm{ng} / \mathrm{ml})$ for 24 hours. Cells were fixed with $3.7 \%$ formalin in PBS, and cell surface ELISAs were performed as previously described (31). Goat polyclonal antibodies specific for JAM-C $(10 \mu \mathrm{g} / \mathrm{ml})$ were used, and the plates were read with an ELISA reader (Bio-Rad) at $450 \mathrm{n} M$.

Immunofluorescence staining. RA ST fibroblasts were plated at 10,000 cells/well in 8-well Labtek chamber slides. After overnight serum starvation, cells were left untreated or were stimulated with $\mathrm{TNF} \alpha(25 \mathrm{ng} / \mathrm{ml})$ for 24 hours. Cells were washed with PBS, fixed with $4 \%$ formalin for 30 minutes at room temperature, washed again with PBS, and fixed with ice-cold methanol for 5 minutes. Blocking was performed by adding 5\% donkey serum in PBS for 1 hour at room temperature. JAM-C-specific primary antibody $(5 \mu \mathrm{g} / \mathrm{ml})$ was added for 1 hour at room temperature in blocking buffer. Following washing, Alexa Fluor 488-conjugated donkey anti-goat antibody $(10 \mu \mathrm{g} / \mathrm{ml})$ was added for 1 hour at room temperature. Cells were washed with PBS, and 4',6-diamidino-2phenylindole (Molecular Probes) nuclear stain was added for 5 minutes at a 1:2,000 dilution. Slides were dehydrated, mounted, and coverslipped. Immunofluorescence staining was detected using an Olympus FV-500 confocal microscope (Olympus America, Melville, NY).

Flow cytometric analysis. U937 cells were collected and washed twice in fluorescence-activated cell sorter (FACS) buffer (PBS/1\% FBS/0.02\% sodium azide) and resuspended at a final concentration of $5 \times 10^{5}$ cells per tube. Cells were then incubated with goat anti-human JAM-A, JAM-B, or JAM-C, at a concentration of $5 \mu \mathrm{g} / \mathrm{ml}$ diluted in FACS buffer, for 30 minutes at $4^{\circ} \mathrm{C}$. Following washing with FACS buffer, cells were resuspended in blocking buffer (30\% donkey serum/ FACS buffer) and incubated for 30 minutes at $4^{\circ} \mathrm{C}$. Cells were then incubated with Alexa Fluor 488 donkey anti-goat secondary antibody at a concentration of $5 \mu \mathrm{g} / \mathrm{ml}$ in blocking buffer for 30 minutes at $4^{\circ} \mathrm{C}$. Secondary antibody with goat IgG was used as a negative control. At the end of the experiment, cells were washed twice with FACS buffer and resuspended in $\mathrm{PBS} / 1 \%$ formaldehyde until analysis. JAM expression on U937 cells was analyzed using a BD FACSAria high-speed cell sorter (BD Biosciences, San Jose, CA), and the percentage of positively stained cells was determined.

In vitro cell adhesion assay. Adhesion of U937 cells to RA ST or OA ST fibroblasts grown to confluence in 96-well plates was tested. RA ST fibroblasts were serum starved overnight, and 1 hour prior to assay, the fibroblasts were preincubated with neutralizing antibodies H33 and F26 against
JAM-C $(25 \mu \mathrm{g} / \mathrm{ml})$ or irrelevant rat IgG controls. U937 cells were left untreated or were stimulated with $100 \mathrm{ng} / \mathrm{ml}$ phorbol myristate acetate (PMA) overnight. The following day, the cells were collected and labeled with Calcein AM fluorescent dye $(5 \mu M)$ (Invitrogen) for 20 minutes. After washing twice, $1 \times 10^{5}$ cells were added to each well and incubated for 20 minutes at room temperature or at $37^{\circ} \mathrm{C}$. At the end of the assay, nonadherent cells were washed off, and fluorescence was measured using a Synergy HT fluorescence plate reader (BioTek Instruments, Winooski, VT).

Stamper-Woodruff assay. In situ adhesion assays were performed as previously described (28). Briefly, frozen RA ST samples were cut into $\sim 5-\mu \mathrm{m}$ sections and incubated with neutralizing antibodies against JAM-C (H33 or F26) or irrelevant IgG control (rat IgG). Neutralizing rabbit antibody against P-selectin was used as a positive control (32). U937 cells were labeled with Calcein AM fluorescent dye $(5 \mu M)$ (Invitrogen) for 20 minutes. After incubation, medium was removed, and $1 \times 10^{5}$ fluorescence-labeled U937 cells were added to all sections and incubated for 1 hour at room temperature. At the end of the experiment, nonadherent cells were washed off. Fluorescent U937 cell adhesion to RA ST was counted by an investigator (BJR) who was blinded with regard to treatment group, using a BX51 Fluorescence Microscope System and DP Manager imaging software (Olympus America)

Leukocyte-RA ST fibroblast transmigration assay. RA ST fibroblasts $\left(1.5 \times 10^{4}\right)$ were grown to confluence in Costar Transwell culture inserts in 24-well tissue culture plates (10-mm diameter polycarbonate membranes with 8 - $\mu \mathrm{m}$ diameter pores) (Corning Costar, Cambridge, MA). At the start of the assay, cultures were washed twice, and RPMI $/ 0.5 \%$ bovine serum albumin was loaded into the lower compartment of each plate with or without SDF- $1 \alpha(200 \mathrm{ng} / \mathrm{ml})$. U937 cells $\left(2 \times 10^{5}\right)$ were added to each filter in the presence of neutralizing anti-JAM-C antibody (H33 or F26) or irrelevant IgG control and incubated for 2 or 4 hours at $37^{\circ} \mathrm{C}$. At the end of the assay, the transmigrated cells were collected from the lower chamber, and their number was determined using a CyQuant cell enumeration kit (Invitrogen).

Statistical analysis. Data were analyzed using Student's $t$-test assuming equal variances. Data are reported as the mean \pm SEM. $P$ values less than 0.05 were considered significant.

\section{RESULTS}

Expression of JAM-C on RA synovial lining cells and ECs. JAM-C expression was determined in ST samples from normal subjects, OA patients, and RA patients using immunohistochemical staining. JAM-C has previously been shown to be expressed in both ST lining cells and ECs in both OA and RA patients by Palmer et al (27); however, JAM-C expression was not quantified in each cell type within the ST samples. As expected, both synovial inflammation and vascularity were significantly enhanced in RA compared with normal ST samples $(P<0.05)$ (data not shown). 


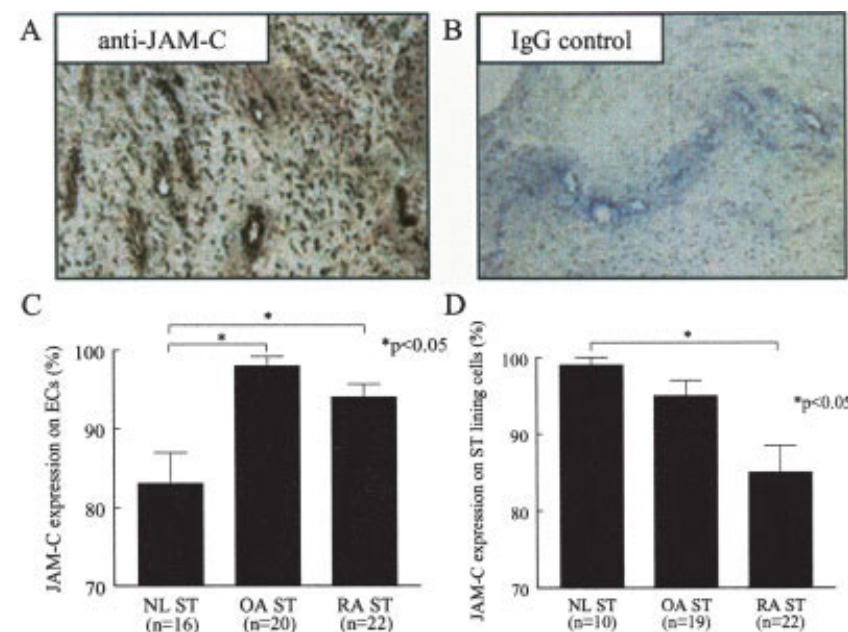

Figure 1. Immunohistologic analysis of junctional adhesion molecule C (JAM-C) in synovial tissue (ST) samples from normal (NL) subjects, patients with osteoarthritis (OA), and patients with rheumatoid arthritis (RA). Frozen sections of normal, OA, and RA ST were stained for JAM-C expression. A and B, Representative photomicrographs of JAM-C (A) and IgG control (B) expression in RA ST (original magnification $\times 400$ ). C, Mean and SEM JAM-C expression in normal ST, OA ST, and RA ST endothelial cells (ECs). JAM-C was more highly expressed in OA ST (98\%) and RA ST (94\%) ECs than in normal ST $(83 \%)$ ECs. D, Mean and SEM JAM-C expression in normal ST, OA ST, and RA ST lining cells. JAM-C was more highly expressed in normal ST (99\%) and OA ST (95\%) lining cells than in RA ST $(85 \%)$ lining cells.

JAM-C expression was analyzed in both ECs and synovial lining cells by a pathologist $(\mathrm{GKH})$ who was blinded with regard to cell type. Figure 1 shows representative photomicrographs of RA ST samples immunostained with JAM-C antibody (Figure 1A) or irrelevant goat IgG (Figure 1B). ECs within both OA and RA ST expressed high levels of JAM-C (mean \pm SEM $98 \pm 1.7 \%$ and $94 \pm 1.7 \%$, respectively), while ECs within normal ST expressed significantly less JAM-C than did ECs within RA ST $(83 \pm 3.9 \%, P<$ 0.05 ) (Figure 1C). In addition, we found that JAM-C was highly expressed by ST lining cells from normal, $\mathrm{OA}$, and RA ST $(99 \pm 1.0 \%, 95 \pm 2.0 \%$, and 85 $\pm 3.5 \%$, respectively), with lining cell expression in normal and OA ST significantly greater than that in RA ST $(P<0.05)$ (Figure 1D).

Expression of JAM-C in RA ST fibroblasts. Previously, JAM-C messenger RNA (mRNA) has been shown to be present in RA ST fibroblasts (27). To determine whether JAM-C was expressed by RA ST fibroblasts at the protein level and whether it was regulated by the proinflammatory cytokine TNF $\alpha$, Western blots were performed. We chose $\mathrm{TNF} \alpha$ to stimulate
RA ST fibroblasts, since TNF $\alpha$ is a pluripotent cytokine important in RA pathogenesis and has been shown to induce RA ST fibroblast surface expression of other adhesion molecules $(33,34)$. Western blotting revealed a $35-\mathrm{kd}$ protein band corresponding to JAM-C; however, no increase in expression was observed following overnight stimulation with $\mathrm{TNF} \alpha$ (Figure $2 \mathrm{~A}$ ).

In addition, we performed a cell surface ELISA to determine if JAM-C was expressed on the surface of RA ST fibroblasts. We found that JAM-C was
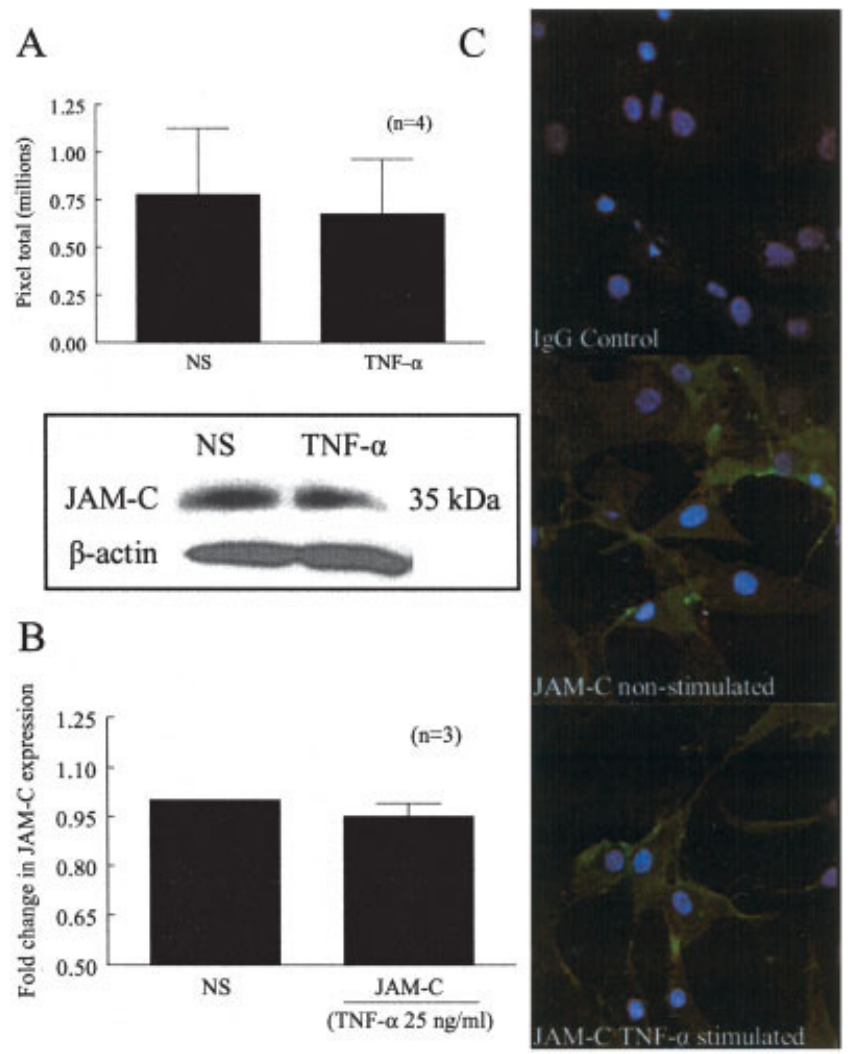

Figure 2. JAM-C expression in RA ST fibroblasts. A, Mean and SEM total number of pixels showing JAM-C expression in RA ST fibroblasts left untreated or cultured with tumor necrosis factor $\alpha(\mathrm{TNF} \alpha)$ for 24 hours. Western blots showed a 35-kd protein band consistent with the molecular weight of JAM-C; however, no differences were observed after stimulation with $\mathrm{TNF} \alpha$. B, Mean and SEM fold change in expression of JAM-C on the cell surface of RA ST fibroblasts left untreated or cultured with $\mathrm{TNF} \alpha$, determined by cell surface enzymelinked immunosorbent assay. Surface expression of JAM-C on RA ST fibroblasts did not change after stimulation with TNF $\alpha$. C, Surface expression and localization of IgG control and JAM-C on RA ST fibroblasts, examined using immunofluorescence and confocal microscopy. JAM-C was expressed throughout the surface, and no difference in expression was noted between adjacent fibroblasts in the presence or absence of TNF $\alpha$. NS = nonstimulated (see Figure 1 for other definitions). 

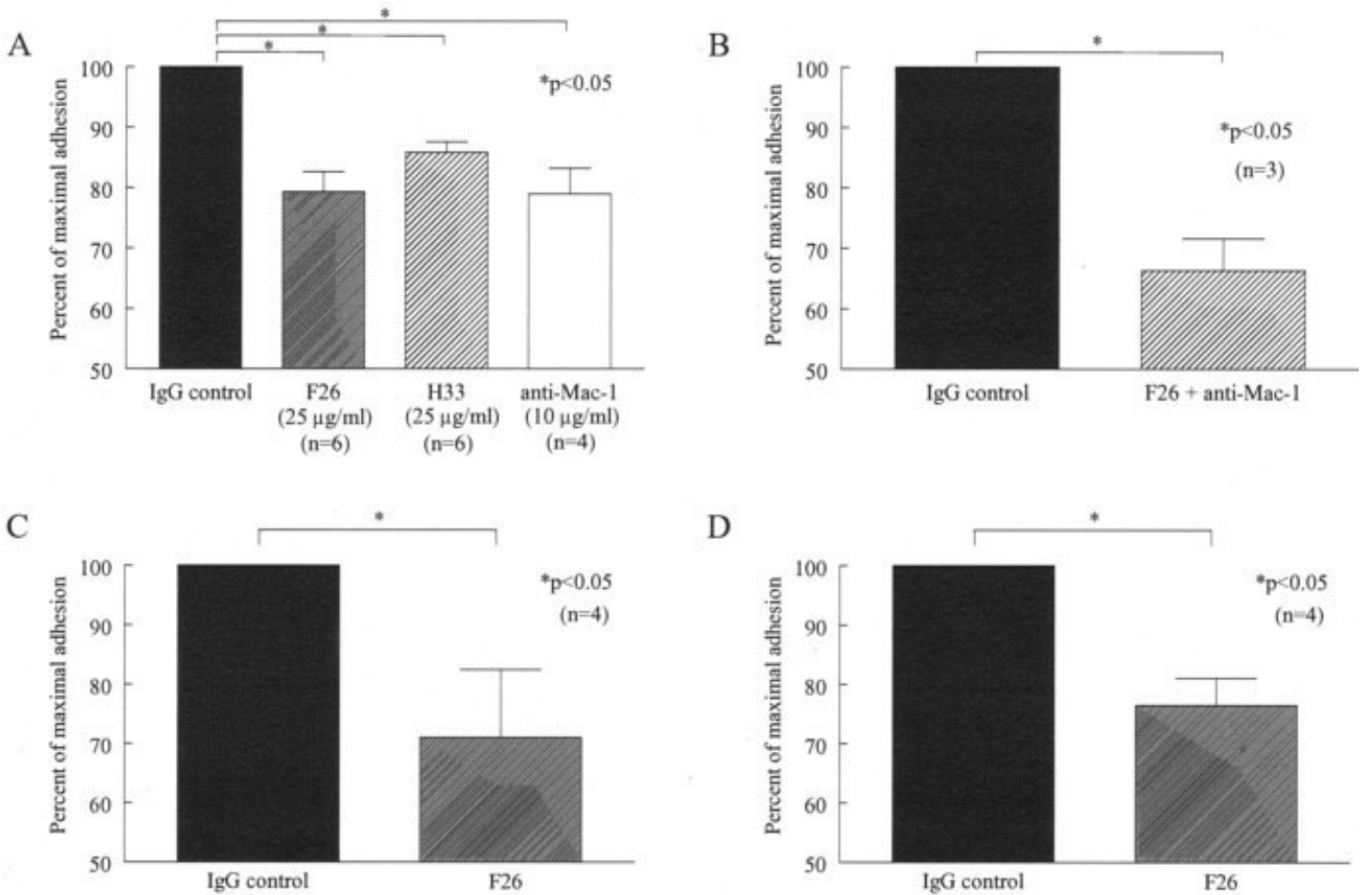

Figure 3. JAM-C mediates adhesion of untreated or phorbol myristate acetate (PMA)-stimulated U937 cells to RA and OA ST fibroblasts. The percentage of maximal adhesion was defined as the number of adherent cells on the ST sections divided by the number of adherent cells on the control sections. A, Inhibition of U937 cell adhesion to RA ST fibroblasts by both of the neutralizing antibodies against JAM-C, F26 (79\% of maximal adhesion) and H33 (86\% of maximal adhesion), and by an anti-Mac-1 antibody (79\% of maximal adhesion). B, Additive inhibitory effect on U937 cell adhesion to RA ST fibroblasts of F26 combined with anti-Mac-1 (66\% of maximal adhesion). C, Inhibition of PMA-stimulated U937 cell adhesion to RA ST fibroblasts at $37^{\circ} \mathrm{C}$ by F26 (71\% of maximal adhesion). D, Inhibition of PMA-stimulated U937 cell adhesion to OA ST fibroblasts at $37^{\circ} \mathrm{C}$ by F26 (77\% of maximal adhesion). Bars show the mean and SEM. See Figure 1 for other definitions.

present on the surface of these cells, and similar to the results of Western blotting, no increase in expression was observed following stimulation with $\mathrm{TNF} \alpha$ (Figure 2B). To visualize JAM-C expression on RA ST fibroblasts, immunofluorescence was performed via confocal microscopy. JAM-C was expressed on the surface of RA ST fibroblasts, but was not confined to cell junctions (Figure 2C). Overnight stimulation with $\mathrm{TNF} \alpha$ or plating on gelatin-coated plates did not result in an increase or redistribution of JAM-C on the cell surface. Similar results were observed with respect to OA ST fibroblasts, as JAM-C was expressed on the cell surface but not confined to the cell surface (data not shown). Moreover, the distribution of JAM-C on the surface of OA ST fibroblasts did not change after stimulation with $\mathrm{TNF} \alpha$ or plating on gelatin-coated wells. Taken together, these results indicate that while JAM-C is present on the cell surface of RA and OA ST fibroblasts, its expression is not regulated by $\mathrm{TNF} \alpha$.

JAM-C mediates U937 cell adhesion to RA ST fibroblasts. To determine the role that JAM-C plays in mediating leukocyte adhesion to RA ST fibroblasts, we performed in vitro adhesion assays. We found that either of the neutralizing anti-JAM-C antibodies, H33 or F26, significantly inhibited adhesion of U937 cells to RA ST fibroblasts, by $14 \%$ and $21 \%$, respectively $(P<0.05$ versus $\operatorname{IgG}$ control) (Figure 3A). Incubation with an antibody against Mac-1 also significantly inhibited U937 cell adhesion to RA ST fibroblasts, by $21 \%(P<0.05$ versus IgG control) (Figure 3A). Moreover, when RA ST fibroblasts were preincubated with a combination of F26 and anti-Mac-1 antibodies, an additive inhibition was observed; adhesion decreased by $34 \%(P<0.05)$ 


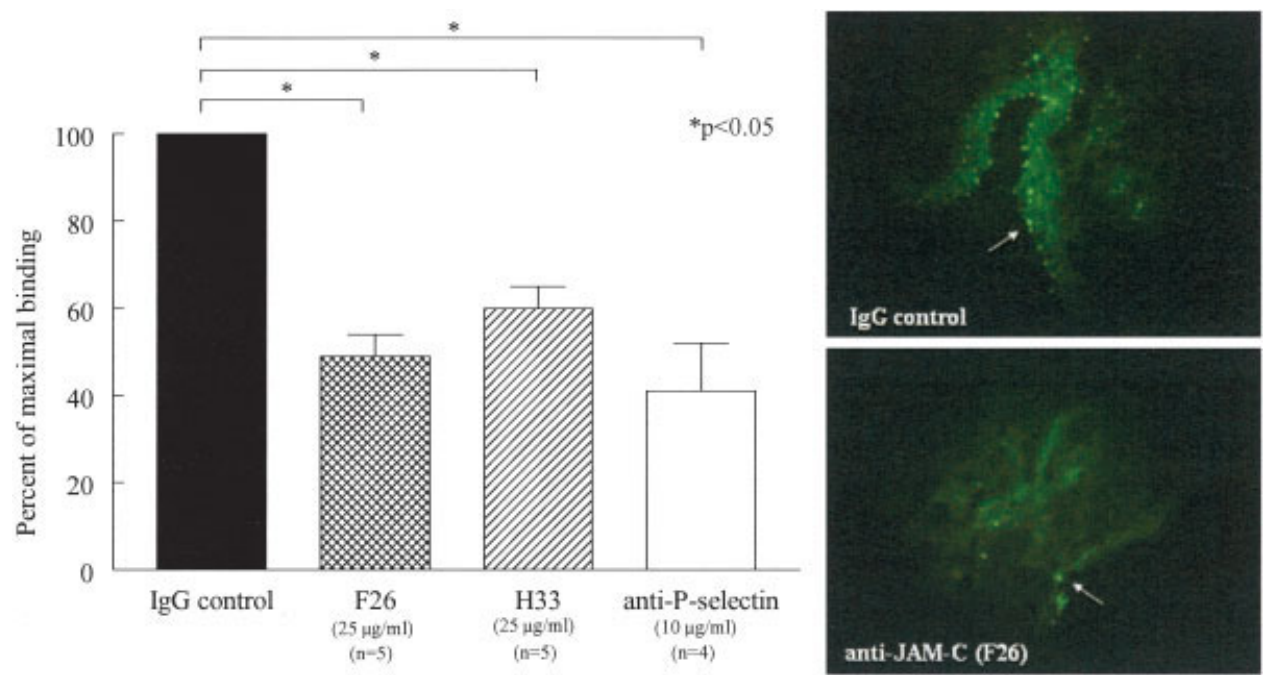

Figure 4. JAM-C mediates adhesion of U937 cells to RA synovium. Stamper-Woodruff in situ assays were performed using frozen RA ST sections and fluorescence-labeled U937 cells. Left, Mean and SEM percentage of maximal binding, defined as the number of adherent cells on the test sections divided by the number of adherent cells on the control sections, of U937 cells by IgG, F26, H33, and anti-P-selectin. U937 cell adhesion was inhibited to a similar degree by both of the anti-JAM-C antibodies, F26 (49\% of maximal binding) and H33 ( $60 \%$ of maximal binding), and by anti-P-selectin (41\% of maximal binding). Right, Most U937 cells adhered to the blood vessels and synovial lining (arrows) (original magnification $\times 400$ ). See Figure 1 for definitions.

(Figure 3B). In addition, the F26 antibody inhibited PMAstimulated U937 cells from binding to RA ST fibroblasts by $29 \%$ ( $P<0.05$ versus $\mathrm{IgG}$ control) (Figure $3 \mathrm{C}$ ).

To see if this effect was specific to RA ST fibroblasts, we performed in vitro adhesion assays between OA ST fibroblasts and U937 cells. We found that the neutralizing anti-JAM-C antibody F26 significantly inhibited adhesion of PMA-stimulated U937 cells to OA ST fibroblasts, by $23 \%$ ( $P<0.05$ versus IgG control), a level similar to that observed with RA ST fibroblasts (Figure 3D). These results suggest that JAM-C plays a role in mediating leukocyte adhesion to RA ST and OA ST, largely through its interaction with Mac-1.

JAM-C mediates U937 cell adhesion to RA synovium. To determine if JAM-C plays a functional role in mediating leukocyte adhesion to RA ST, we performed in situ cell adhesion assays. We found that myeloid U937 cells preferentially adhered to the synovial lining, which contains myeloid and fibroblast cells, and blood vessels (Figure 4). As a positive control, we used a neutralizing antibody against P-selectin, since $\mathrm{P}$-selectin has previously been shown to meditate human monocyte adhesion to RA ST (32). Anti-P-selectin antibody inhibited U937 adhesion by 59\% (41\% of maximal binding) $(P<$ 0.05 versus IgG control). Importantly, anti-JAM-C neutralizing antibodies H33 and F26 decreased U937 cell adhesion to RA ST by $40 \%$ ( $60 \%$ of maximal binding) and $51 \%$ (49\% of maximal binding), respectively ( $P<$ 0.05 versus IgG control, for both $\mathrm{H} 33$ and F26).

Both anti-JAM-C antibodies were examined due to their different binding specificities. Previously, both H33 and F26 have been shown to neutralize JAM-C homophilic interactions, but only $\mathrm{H} 33$ has been shown to inhibit JAM-B-JAM-C heterophilic interactions $(8,28)$. Since there was no significant difference between the ability of the anti-P-selectin antibody and the antiJAM-C antibodies to inhibit U937 cell adhesion, these results indicated that JAM-C was an important mediator of leukocyte adhesion to inflamed RA synovium.

JAM-C mediates U937 cell retention in a model of myeloid RA ST fibroblast transmigration. To further study the functional role of JAM-C in RA ST, we performed myeloid RA ST fibroblast transmigration assays. Interestingly, incubation with either H33 or F26 resulted in increased U937 cell migration at 2 hours, by 1.4-fold and 1.2-fold, respectively $(P<0.05$ versus IgG control, for both H33 and F26) (Figure 5). This migration persisted at 4 hours, with a 1.3 -fold increase in migration following incubation with $\mathrm{H} 33(P<0.05$ versus $\operatorname{IgG}$ control) and a 1.2-fold increase following incubation with F26. These results further suggest that JAM-C normally plays a role in the retention of leukocytes in the RA synovium, preventing egress of leuko- 


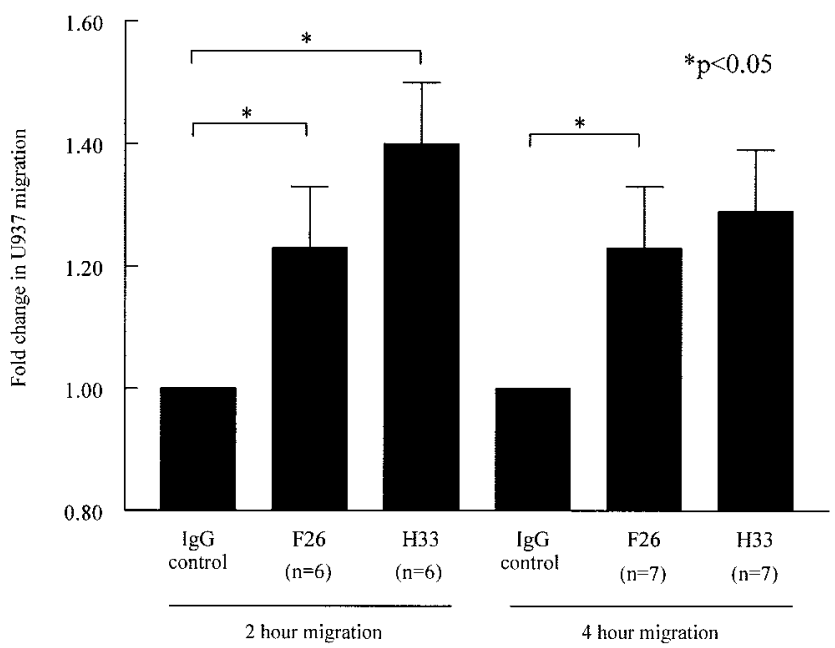

Figure 5. JAM-C mediates U937 cell adhesion in a model of myeloid RA ST fibroblast transmigration. U937 cell migration through RA ST fibroblast monolayers grown on Transwell inserts, in response to incubation with stromal cell-derived factor $1 \alpha$ for 2 or 4 hours, was studied. Both neutralizing anti-JAM-C antibodies, F26 and H33, enhanced migration of U937 cells through the fibroblast monolayer. Bars show the mean and SEM fold change in migrated cells in test wells compared with migration in $\operatorname{IgG}$ control-treated wells. See Figure 1 for definitions.

cytes through the fibroblast monolayer, an effect which can be reversed by anti-JAM-C antibodies.

Expression of JAM-A, but not JAM-B or JAM-C, on U937 cells. Previous studies have demonstrated that the JAM-C ligand Mac-1 is expressed on monocytes and U937 cells (35). Therefore, we wanted to determine if JAM-B, another ligand for JAM-C, is expressed on the surface of U937 cells. Only JAM-A was found to be expressed on the surface of U937 cells (Figure 6). Interestingly, no JAM-B or JAM-C was detected, indicating that neither homophilic JAM-C nor heterophilic JAM-B-JAM-C interactions mediate U937 adhesion to RA ST or OA ST fibroblasts.

\section{DISCUSSION}

In the current study, we have shown that JAM-C is expressed on both ECs and lining cells in normal, OA, and RA ST. While Palmer et al (27) showed that JAM-C is expressed in RA ST and OA ST (27), we found that JAM-C was more highly expressed in ECs in RA and OA ST than ECs in normal ST. In contrast, Ludwig et al (23) have shown that in a mouse model of contact dermatitis, levels of JAM-C mRNA and protein in ECs were similar in normal skin and inflamed skin (23). However, JAM-C has been shown to be overexpressed in both smooth muscle cells and endothelium during arteriosclerosis and to have increasing expression with lesion progression (36). Similarly, JAM-C was shown to be up-regulated in ECs in a mouse model of acute pancreatitis compared with control animals (25). Taken together, these results suggest that JAM-C is upregulated on ECs in various inflammatory states.

After finding JAM-C expression in OA ST and RA ST lining cells, we wanted to determine if JAM-C was expressed in OA ST and RA ST fibroblasts and where it was localized in these cells. Recently, Palmer et al (27) demonstrated that JAM-C mRNA was expressed in RA ST fibroblasts. Our results confirmed these findings at the protein level and demonstrated that JAM-C expression was not dependent on the proinflammatory cytokine TNF $\alpha$. Moreover, we showed that JAM-C is expressed on the surface of RA ST fibroblasts and that its expression is not limited to cell-cell contact points, but rather distributed across the cell surface. Furthermore, the distribution pattern of JAM-C in RA ST fibroblasts was not altered by the addition of TNF $\alpha$ or by plating the cells in the presence of a polarizing extracellular matrix molecule. We observed a similar expression pattern for OA ST fibroblasts.

JAM-C has previously been shown to be expressed on the surface of human dermal, lung, and corneal fibroblasts (37). Morris et al (37) found that under quiescent conditions, JAM-C localizes to the adherens-like junctions between adjacent fibroblasts. Under inflammatory conditions, JAMs redistribute on

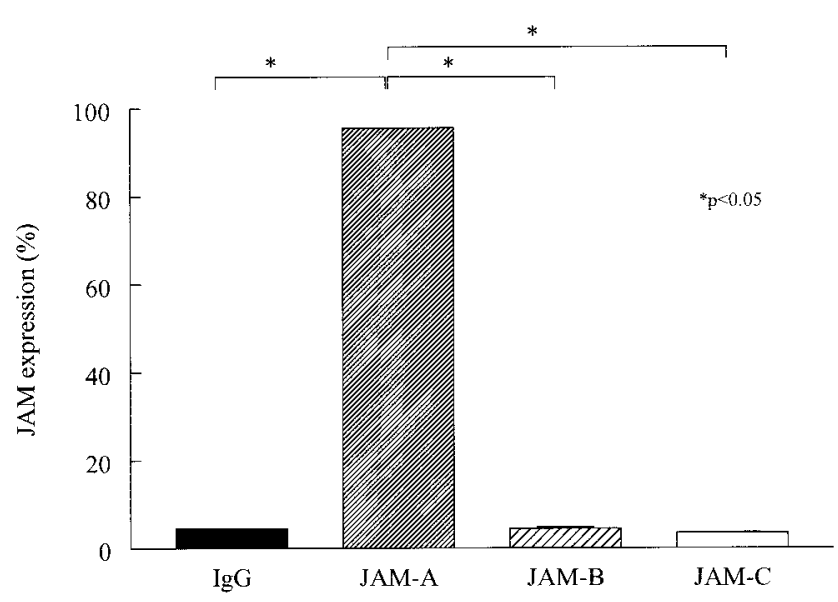

Figure 6. Mean percentage of U937 cells expressing junctional adhesion molecule A (JAM-A), JAM-B, and JAM-C. Flow cytometric analysis was performed on U937 cells immunostained with antibodies against JAM-A, JAM-B, or JAM-C, or IgG control. U937 cells expressed JAM-A, but not JAM-B or JAM-C. 
ECs from cell contact points to the cell surface (38). JAM-C has been shown to be partially redistributed to the cell surface and away from inter-EC contacts following stimulation with oxidized lipoproteins (36). Therefore, the distribution pattern of JAM-C in OA and RA ST fibroblasts suggests that it may play a role in both fibroblast junctional stability and in mediating leukocyte retention, rather than merely stabilizing fibroblast junctions.

Based on the observed expression of JAM-C in OA ST and RA ST, as well as OA ST and RA ST fibroblasts, we sought to determine if JAM-C played a role in mediating leukocyte adhesion to the RA synovium. Previous studies have demonstrated that JAMs play a role in binding leukocyte surface integrins $(14,17,18)$. Liberation of JAM-C from junctional complexes on ECs can lead to apical expression of JAM-C and possible interaction with Mac-1 (28). Under proatherosclerotic conditions, JAM-C is redistributed from the EC junctional complex to the cell surface, which could lead to additional adhesion of leukocytes, thus potentiating the disease (36). Under these conditions, myeloid THP-1 adhesion to ECs and transmigration of THP-1 cells through ECs was increased 2-fold (36). In addition, platelet JAM-C has been shown to interact with the leukocyte cell surface integrin Mac-1 to facilitate firm adhesion between platelets and leukocytes (18). Those investigators also demonstrated that U937 cells, which express Mac-1, bind purified and immobilized JAM-C, as well as JAM-C-transfected Chinese hamster ovary cells (18).

In the present study, we showed JAM-Cdependent U937 cell adhesion to OA ST and RA ST fibroblasts and RA synovial sections. Neutralizing antiJAM-C antibodies, H33 and F26, and neutralizing antiMac-1 antibody reduced adhesion between U937 cells and RA ST fibroblasts to a similar degree. When the F26 anti-JAM-C antibody was added to the anti-Mac-1 antibody, the combination yielded an additive inhibitory effect on adhesion. This indicates that JAM-C mediates adhesion of U937 myeloid cells to RA ST fibroblasts in part through its interaction with the integrin Mac-1.

In addition to mediating leukocyte adhesion, JAMs play a primary role in the transmigration of leukocytes across the endothelium. JAM-C has been shown to promote lymphocyte and neutrophil transendothelial migration $(24,39)$. Under inflammatory conditions, JAM-C has been shown to modulate the influx of leukocytes through the endothelium (26). In a model of acute pulmonary inflammation, leukocyte transendothe- lial migration was increased in mice overexpressing JAM-C specifically on their ECs (26). Moreover, JAM-C has recently been shown to regulate unidirectional monocyte transendothelial migration (40). Bradfield et al (40) showed that blockade of JAM-B-JAM-C interactions reduced monocyte numbers in extravascular tissue by promoting increased reverse transmigration, rather than by reducing transendothelial migration. However, JAM-C was not shown to play a role in neutrophil transendothelial migration under shear flow conditions (41).

In addition to transendothelial migration, JAM-C has been shown to play a role in transepithelial migration (19). Blocking JAM-C, by either monoclonal antibody or soluble recombinant JAM-C, inhibited the rate of polymorphonuclear cell transepithelial migration (19). However, in our study, we found that the addition of anti-JAM-C antibodies resulted in an increase in U937 cell migration through an RA ST fibroblast monolayer. It may be that RA ST fibroblasts are not polarized cells and do not form tight junctions like ECs and epithelial cells. Moreover, we have shown that JAM-C is primarily located at the cell surface of RA ST fibroblasts and is not selectively localized at adhesion points between adjacent fibroblasts. Therefore, we postulate that JAM-C normally plays a role in leukocyte retention with the RA synovium.

Our study was designed to determine JAM-C expression in the synovium and to determine the role of JAM-C in RA ST. We have shown that JAM-C is highly expressed in OA and RA ST lining cells and ECs and on the surface of OA and RA ST fibroblasts. Importantly, we have demonstrated that JAM-C modulates the adhesion to and retention of leukocytes in the RA synovium. We therefore propose that JAM-C plays dual roles in mediating arthritis. As was shown previously, JAM-C on the surface of ECs mediates neutrophil ingress into the arthritic synovium, and we now propose that JAM-C on the surface of RA ST fibroblasts mediates the retention of leukocytes in RA synovium. Taken together, these results demonstrate the importance of JAM-C in RA and suggest that targeting JAM-C may be important in combating RA.

\section{AUTHOR CONTRIBUTIONS}

Dr. Koch had full access to all of the data in the study and takes responsibility for the integrity of the data and the accuracy of the data analysis.

Study design. Rabquer, Pakozdi, Imhof, Koch.

Acquisition of data. Rabquer, Pakozdi, Michel, Gujar, Haines. Analysis and interpretation of data. Rabquer, Pakozdi, Gujar, Haines, Koch. 
Manuscript preparation. Rabquer, Imhof, Koch. Statistical analysis. Rabquer.

\section{REFERENCES}

1. Szekanecz Z, Szegedi G, Koch AE. Cellular adhesion molecules in rheumatoid arthritis: regulation by cytokines and possible clinical importance. J Investig Med 1996;44:124-35.

2. Johnson BA, Haines GK, Harlow LA, Koch AE. Adhesion molecule expression in human synovial tissue. Arthritis Rheum 1993;36:137-46.

3. Van Dinther-Janssen AC, Kraal G, van Soesbergen RM, Scheper RJ, Meijer CJ. Immunohistological and functional analysis of adhesion molecule expression in the rheumatoid synovial lining layer: implications for synovial lining cell destruction. J Rheumatol 1994;21:1998-2004.

4. Martin-Padura I, Lostaglio S, Schneemann M, Williams L, Romano M, Fruscella P, et al. Junctional adhesion molecule, a novel member of the immunoglobulin superfamily that distributes at intercellular junctions and modulates monocyte transmigration. J Cell Biol 1998;142:117-27.

5. Cunningham SA, Arrate MP, Rodriguez JM, Bjercke RJ, Vanderslice P, Morris AP, et al. A novel protein with homology to the junctional adhesion molecule: characterization of leukocyte interactions. J Biol Chem 2000;275:34750-6.

6. Palmeri D, van Zante A, Huang CC, Hemmerich S, Rosen SD. Vascular endothelial junction-associated molecule, a novel member of the immunoglobulin superfamily, is localized to intercellular boundaries of endothelial cells. J Biol Chem 2000;275:19139-45.

7. Arrate MP, Rodriguez JM, Tran TM, Brock TA, Cunningham SA. Cloning of human junctional adhesion molecule 3 (JAM3) and its identification as the JAM2 counter-receptor. J Biol Chem 2001; 276:45826-32.

8. Aurrand-Lions M, Duncan L, Ballestrem C, Imhof BA. JAM-2, a novel immunoglobulin superfamily molecule, expressed by endothelial and lymphatic cells. J Biol Chem 2001;276:2733-41.

9. Hirabayashi S, Tajima M, Yao I, Nishimura W, Mori H, Hata Y. JAM4, a junctional cell adhesion molecule interacting with a tight junction protein, MAGI-1. Mol Cell Biochem 2003;23:4267-82.

10. Moog-Lutz C, Cave-Riant F, Guibal FC, Breau MA, Di Gioia Y, Couraud PO, et al. JAML, a novel protein with characteristics of a junctional adhesion molecule, is induced during differentiation of myeloid leukemia cells. Blood 2003;102:3371-8.

11. Weber C, Fraemohs L, Dejana E. The role of junctional adhesion molecules in vascular inflammation. Nat Rev Immunol 2007;7: 467-77.

12. Kostrewa D, Brockhaus M, D'Arcy A, Dale GE, Nelboeck P, Schmid G, et al. X-ray structure of junctional adhesion molecule: structural basis for homophilic adhesion via a novel dimerization motif. EMBO J 2001;20:4391-8.

13. Prota AE, Campbell JA, Schelling P, Forrest JC, Watson MJ, Peters TR, et al. Crystal structure of human junctional adhesion molecule 1: implications for reovirus binding. Proc Natl Acad Sci U S A 2003;100:5366-71.

14. Ostermann G, Weber KS, Zernecke A, Schroder A, Weber C. JAM-1 is a ligand of the $\beta_{2}$ integrin LFA- 1 involved in transendothelial migration of leukocytes. Nat Immunol 2002;3:151-8.

15. Naik MU, Mousa SA, Parkos CA, Naik UP. Signaling through JAM- 1 and $\alpha v \beta 3$ is required for the angiogenic action of bFGF: dissociation of the JAM-1 and $\alpha \mathrm{v} \beta 3$ complex. Blood 2003;102: 2108-14.

16. Naik MU, Naik UP. Junctional adhesion molecule-A-induced endothelial cell migration on vitronectin is integrin $\alpha \mathrm{v} \beta 3$ specific. J Cell Sci 2006;119(Pt 3):490-9.

17. Cunningham SA, Rodriguez JM, Arrate MP, Tran TM, Brock TA.
JAM2 interacts with $\alpha 4 \beta 1$ : facilitation by JAM3. J Biol Chem 2002;277:27589-92.

18. Santoso S, Sachs UJ, Kroll H, Linder M, Ruf A, Preissner KT, et al. The junctional adhesion molecule 3 (JAM-3) on human platelets is a counterreceptor for the leukocyte integrin Mac-1. J Exp Med 2002;196:679-91.

19. Zen K, Babbin BA, Liu Y, Whelan JB, Nusrat A, Parkos CA. JAM-C is a component of desmosomes and a ligand for CD11b/ CD18-mediated neutrophil transepithelial migration. Mol Biol Cell 2004:15:3926-37.

20. Corada M, Chimenti S, Cera MR, Vinci M, Salio M, Fiordaliso F, et al. Junctional adhesion molecule-A-deficient polymorphonuclear cells show reduced diapedesis in peritonitis and heart ischemia-reperfusion injury. Proc Natl Acad Sci U S A 2005;102: 10634-9.

21. Del Maschio A, De Luigi A, Martin-Padura I, Brockhaus M, Bartfai T, Fruscella P, et al. Leukocyte recruitment in the cerebrospinal fluid of mice with experimental meningitis is inhibited by an antibody to junctional adhesion molecule (JAM). J Exp Med 1999;190:1351-6.

22. Khandoga A, Kessler JS, Meissner H, Hanschen M, Corada M, Motoike T, et al. Junctional adhesion molecule-A deficiency increases hepatic ischemia-reperfusion injury despite reduction of neutrophil transendothelial migration. Blood 2005;106:725-33.

23. Ludwig RJ, Zollner TM, Santoso S, Hardt K, Gille J, Baatz H, et al. Junctional adhesion molecules (JAM)-B and -C contribute to leukocyte extravasation to the skin and mediate cutaneous inflammation. J Invest Dermatol 2005;125:969-76.

24. Chavakis T, Keiper T, Matz-Westphal R, Hersemeyer K, Sachs UJ, Nawroth PP, et al. The junctional adhesion molecule-C promotes neutrophil transendothelial migration in vitro and in vivo. J Biol Chem 2004;279:55602-8.

25. Vonlaufen A, Aurrand-Lions M, Pastor CM, Lamagna C, Hadengue $\mathrm{A}$, Imhof $\mathrm{BA}$, et al. The role of junctional adhesion molecule C (JAM-C) in acute pancreatitis. J Pathol 2006;209:540-8.

26. Aurrand-Lions M, Lamagna C, Dangerfield JP, Wang S, Herrera $\mathrm{P}$, Nourshargh $\mathrm{S}$, et al. Junctional adhesion molecule-C regulates the early influx of leukocytes into tissues during inflammation. J Immunol 2005;174:6406-15.

27. Palmer G, Busso N, Aurrand-Lions M, Talabot-Ayer D, ChobazPeclat V, Zimmerli C, et al. Expression and function of junctional adhesion molecule-C in human and experimental arthritis. Arthritis Res Ther 2007;9:R65.

28. Lamagna C, Meda P, Mandicourt G, Brown J, Gilbert RJ, Jones EY, et al. Dual interaction of JAM-C with JAM-B and $\alpha_{\mathrm{M}} \beta_{2}$ integrin: function in junctional complexes and leukocyte adhesion. Mol Biol Cell 2005;16:4992-5003.

29. Woods JM, Mogollon A, Amin MA, Martinez RJ, Koch AE. The role of COX-2 in angiogenesis and rheumatoid arthritis. Exp Mol Pathol 2003;74:282-90.

30. Laemmli UK. Cleavage of structural proteins during the assembly of the head of bacteriophage T4. Nature 1970;227:680-5.

31. Amin MA, Haas CS, Zhu K, Mansfield PJ, Kim MJ, Lackowski NP, et al. Migration inhibitory factor up-regulates vascular cell adhesion molecule-1 and intercellular adhesion molecule-1 via Src, PI3 kinase, and NF $\kappa$ B. Blood 2006;107:2252-61.

32. Grober JS, Bowen BL, Ebling H, Athey B, Thompson CB, Fox DA, et al. Monocyte-endothelial adhesion in chronic rheumatoid arthritis: in situ detection of selectin and integrin-dependent interactions. J Clin Invest 1993;91:2609-19.

33. Croft D, McIntyre P, Wibulswas A, Kramer I. Sustained elevated levels of VCAM-1 in cultured fibroblast-like synoviocytes can be achieved by TNF- $\alpha$ in combination with either IL-4 or IL-13 through increased mRNA stability. Am J Pathol 1999;154: 1149-58.

34. Morales-Ducret J, Wayner E, Elices MJ, Alvaro-Garcia JM, 
Zvaifler NJ, Firestein GS. $\alpha 4 / \beta 1$ integrin (VLA-4) ligands in arthritis vascular cell adhesion molecule- 1 expression in synovium and on fibroblast-like synoviocytes. J Immunol 1992;149:1424-31.

35. Shelley CS, Arnaout MA. The promoter of the CD11b gene directs myeloid-specific and developmentally regulated expression. Proc Natl Acad Sci U S A 1991;88:10525-9.

36. Keiper T, Al-Fakhri N, Chavakis E, Athanasopoulos AN, Isermann B, Herzog S, et al. The role of junctional adhesion molecule-C (JAM-C) in oxidized LDL-mediated leukocyte recruitment. FASEB J 2005;19:2078-80.

37. Morris AP, Tawil A, Berkova Z, Wible L, Smith CW, Cunningham SA. Junctional adhesion molecules (JAMs) are differentially expressed in fibroblasts and co-localize with ZO-1 to adherens-like junctions. Cell Commun Adhes 2006;13:233-47.
38. Weber C, Fraemohs L, Dejana E. The role of junctional adhesion molecules in vascular inflammation. Nat Rev Immunol 2007;7: 467-77.

39. Johnson-Leger CA, Aurrand-Lions M, Beltraminelli N, Fasel N, Imhof BA. Junctional adhesion molecule-2 (JAM-2) promotes lymphocyte transendothelial migration. Blood 2002;100:2479-86.

40. Bradfield PF, Scheiermann C, Nourshargh S, Ody C, Luscinskas FW, Rainger GE, et al. JAM-C regulates unidirectional monocyte transendothelial migration in inflammation. Blood 2007;110: 2545-55.

41. Sircar M, Bradfield PF, Aurrand-Lions M, Fish RJ, Alcaide P, Yang L, et al. Neutrophil transmigration under shear flow conditions in vitro is junctional adhesion molecule-C independent. J Immunol 2007;178:5879-87. 